\title{
The Localized Enhancement of Surface Plasmon Standing Waves Interacting With Single Nanoparticles
}

hongyao liu ( $\square$ liuhongyao@ime.ac.cn )

CAS Institute of Microelectronics: Institute of Microelectronics pf Chinese Academy of Sciences https://orcid.org/0000-0002-2295-4441

\section{Xuqing Sun}

CAS Institute of Microelectronics: Institute of Microelectronics pf Chinese Academy of Sciences

\section{Xue Wang}

CAS Institute of Microelectronics: Institute of Microelectronics pf Chinese Academy of Sciences

\section{Fei Wang}

CAS Institute of Microelectronics: Institute of Microelectronics pf Chinese Academy of Sciences

\section{Chang Wang}

Institute of Microelectronics

\section{Xiaojuan Sun}

CAS Institute of Microelectronics: Institute of Microelectronics pf Chinese Academy of Sciences

Xinchao Lu

CAS Institute of Microelectronics: Institute of Microelectronics pf Chinese Academy of Sciences

\section{Chengjun Huang}

CAS Institute of Microelectronics: Institute of Microelectronics pf Chinese Academy of Sciences

\section{Research Article}

Keywords: Surface plasmon polariton, Standing wave, Nanoparticles, Sensitivity, Dipole mode

Posted Date: March 9th, 2021

DOl: https://doi.org/10.21203/rs.3.rs-254097/v1

License: (c) (i) This work is licensed under a Creative Commons Attribution 4.0 International License. Read Full License 


\title{
The localized enhancement of surface plasmon standing waves interacting with single nanoparticles
}

\author{
Hongyao Liu ${ }^{1}$ - Xuqing Sun ${ }^{1,2}$ - Xue Wang1,2 Fei \\ Wang $^{1,2}$ • Chang Wang ${ }^{1,2}$ - Xiaojuan Sun ${ }^{1,2}$ - Xinchao \\ Lu $^{1}$ - Chengjun Huang ${ }^{1,2}$
}

\begin{abstract}
Real-time, high-sensitivity, and label-free detection to single nanoparticles has been achieved via visualizing the interaction between surface plasmon polaritons (SPPs) and nanoparticles, which is widely applied to chemistry and biology. In this work, aiming to enhance the detection sensitivity to nanoparticles, we explore the interaction of SPP standing waves with single nanoparticles. Compared with SPPs, the inhomogeneous fields of SPP standing waves modulate charge distributions around the particle and excite different electric dipole modes that tailor localized enhancements. For nanoparticles situating at electric antinodes of SPP standing waves, a vertical electric dipole is excited and highdensity charges are stimulated around nanoparticle-film nanocavities, leading to further increased localized enhancement. The localized enhancement experiences more increase with smaller particle size, lower dielectric constant of surrounding medium, and lower particle refractive index. Via tailoring the localized enhancement by SPP standing waves, the sensitivity of SPP microscopy can be improved, which would broaden its applications on nanotechnology, biomedicine, and environmental monitoring.
\end{abstract}

Keywords Surface plasmon polariton. Standing wave . Nanoparticles. Sensitivity . Dipole mode

\section{Introduction}

Real-time and label-free detection to single nanoparticles is highly desirable in various fields ranging from material science, biological science to environmental monitoring. Most detection methods cannot meet the demands of high sensitivity and fast speed simultaneously [1], which makes the label-free and fast detection to single nanoparticles difficult. Surface plasmon polaritons (SPPs) are evanescent waves propagating along the metal-dielectric interface [2,3]. Benefitting from the SPPs' localized enhancement in subwavelength scale, SPP microscopy has been used to achieve real-time, high sensitivity, label-free detection to single nanoparticles [4]. Single nanoparticles induce the localized enhancement of SPPs, and transforms to far field via leakage radiation for fast and label-free detection $[5,6]$. This method has been widely applied to chemistry and biology, such as bidirectional electron transfers visualizing [7], local activation energy barrier measuring [8], thermal hysteresis imaging of single spin-crossover nanoparticles [9], and single liposomes and virus detection [10-12].

SPP standing waves are generated by the interference between two counter propagating SPPs [13-15]. People had used SPP standing waves as structured illumination to excite fluorophores [16-18], and achieved the higher sensitivity by detecting the fluorescent beads. Different from the SPP standing waves interacting with the fluorescent beads, the sensitivity

Corresponding author Xinchao Lu luxinchao@ime.ac.cn

Chengjun Huang huangchengjun@ime.ac.cn

${ }^{1}$ Institute of Microelectronics of Chinese Academy of Sciences, Beijing 100029, China

${ }^{2}$ University of Chinese Academy of Sciences, Beijing 100049, China 
of label-free detection to single nanoparticles is determined by evaluating the localized enhancement $[4,6]$. By using the SPP standing waves to obtain stronger localized enhancement, the detection sensitivity could be improved. As the SPP fields possess constant intensity along metal-dielectric interface, the localized enhancement mainly depends on nanoparticle characteristics and environmental refractive index [19-21]. Comparatively, the SPP standing waves possess amplitude changing periodically along the metal-dielectric interface. The localized enhancement of SPP standing waves induced by single nanoparticles becomes complicated due to the periodic alteration of SPP intensities.

In this paper, we explore the localized enhancement of single nanoparticles interacting with the SPP standing waves. After theoretically analyzed the properties of SPP standing waves, we discuss the SPP standing waves interacting with single nanoparticles via simulations. It shows that different electric dipole modes are excited by situating the particles at different positions of SPP standing waves, leading to various localized enhancements. The vertical electric dipoles are excited for the nanoparticles at the antinodes. Compared with SPPs, the charge density near nanoparticle-metal film nanocavity is evidently higher, leading to increased localized enhancement. Smaller particle size, lower dielectric constant of surrounding medium, and lower particle refractive index contribute to more increased localized enhancement. By increasing the localized enhancement via the SPP standing waves, this work presents a resolution to improve the sensitivity of label-free SPP microscopy.

\section{Characteristics of SPP standing waves}

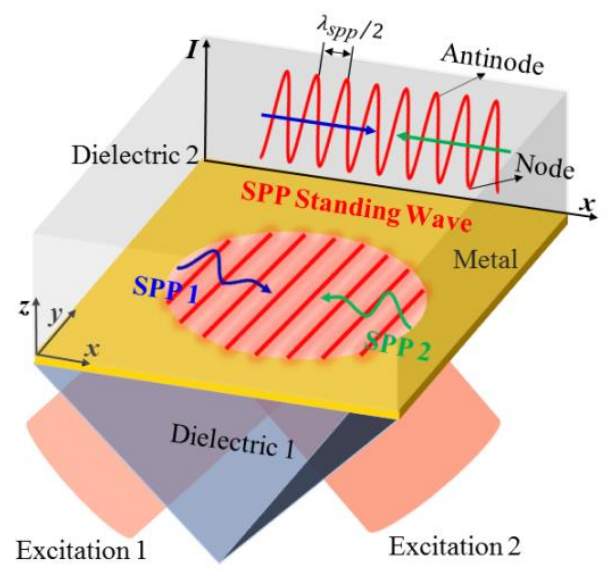

Fig. 1. The model of SPP standing wave excitation in Kretschmman configuration with dielectric-metal-dielectric system

The SPP standing waves are generated by interference of two propagating SPPs with same propagation constant travelling in opposite directions. Figure 1 depicts the model of SPP standing waves excited by Kretschmman configuration in dielectric-metal-dielectric system, which includes a high index medium (dielectric 1) with dielectric constant $\varepsilon_{1}$, a metal slab with thickness $d_{m}$ and dielectric constant $\varepsilon_{m}$, and a low index medium (dielectric 2) with dielectric constant $\varepsilon_{2}$. Illuminating the metal slab with a TM incidence from dielectric 1 under excitation angle $\theta$, SPPs can be excited at the metal-dielectric 2 interface. The vector fields of SPP waves in dielectric $2, \vec{E}_{s p p}=\left(E_{x}, 0, E_{z}\right)$ and $\vec{H}_{s p p}=\left(0, H_{y}, 0\right)$ are as followings [2]: 


$$
\left\{\begin{array}{l}
E_{x}=\frac{B \sqrt{I_{0}} k_{z} \exp \left(-\left|k_{z}\right| z\right)}{\omega \varepsilon_{0} \varepsilon_{2}} \exp \left(i k_{x} x+i \varphi+\frac{i 3 \pi}{2}\right) \\
E_{z}=\frac{B \sqrt{I_{0}} k_{x} \exp \left(-\left|k_{z}\right| z\right)}{\omega \varepsilon_{0} \varepsilon_{2}} \exp \left(i k_{x} x+i \varphi+i \pi\right) \\
H_{y}=B \sqrt{I_{0}} \exp \left(-\left|k_{z}\right| z\right) \exp \left(i k_{x} x+i \varphi\right)
\end{array}\right.
$$

in which $B$ is a constant depending on excitation configuration, $I_{0}$ represents incident light intensity, $k_{x}$ and $k_{z}$ are the tangential and normal wavevectors of SPPs in dielectric 2, given by $k_{x}=\left|k_{0}\right| \sqrt{\varepsilon_{1}} \sin \theta \cos \phi$, and $k_{z}=-\left|k_{0}\right| \sqrt{\varepsilon_{2}-\varepsilon_{1} \sin ^{2} \theta}$, $\theta=\arcsin \sqrt{\operatorname{Re}\left(\varepsilon_{m}\right) \varepsilon_{2} /\left[\varepsilon_{1} \varepsilon_{2}+\varepsilon_{1} \operatorname{Re}\left(\varepsilon_{m}\right)\right]}$ is the incident angle, $\phi=0$ or $\pi$ represents the azimuthal angle in $x y$ plane, $\varphi$ is the initial phase, $\omega$ is the angular frequency. By using two coherent TM incidences with same intensities and incident angles satisfying $\phi_{1}=0$, $\phi_{2}=\pi, \theta_{1}=\theta_{2}=\theta$, two counter propagating SPPs are excited at the metal-dielectric 2 interface. The two SPP waves interfere in the overlap region and form SPP standing waves, whose field distributions are $\vec{E}_{\text {sppsw }}=\left(E_{x}^{\prime}, 0, E_{z}^{\prime}\right)$ and $\vec{H}_{\text {sppsw }}=\left(0, H_{y}^{\prime}, 0\right)$, which can be expressed as

$$
\left\{\begin{array}{l}
E_{x}^{\prime}=\frac{2 B \sqrt{I_{1}} k_{z} \exp \left(-\left|k_{z}\right| z\right)}{\omega \varepsilon_{0} \varepsilon_{2}} \cos \left(k_{x} x+\Delta \varphi\right) \exp \left(i \varphi^{\prime}+i \frac{3 \pi}{2}\right) \\
E_{z}^{\prime}=\frac{2 B \sqrt{I_{1}} k_{x} \exp \left(-\left|k_{z}\right| z\right)}{\omega \varepsilon_{0} \varepsilon_{2}} \cos \left(k_{x} x+\Delta \varphi-\frac{\pi}{2}\right) \exp \left(i \varphi^{\prime}-i \frac{\pi}{2}\right) \\
H_{y}^{\prime}=2 B \sqrt{I_{1}} \exp \left(-\left|k_{z}\right| z\right) \cos \left(k_{x} x+\Delta \varphi\right) \exp \left(i \varphi^{\prime}+\frac{i \pi}{2}\right)
\end{array}\right.
$$

where $I_{1}$ represents intensity of two incident lights, $\Delta \varphi=\left(\varphi_{1}-\varphi_{2}\right) / 2, \varphi^{\prime}=\left(\varphi_{1}+\varphi_{2}\right) / 2$, $\varphi_{1}$ and $\varphi_{2}$ are the initial phases of two incident lights respectively.

Some characteristics of SPP standing waves are extracted from Eqs. (2). Similar with SPPs, the SPP standing waves are also evanescent waves, whose fields bound to the metaldielectric interface and decay length is approximately equal to that of SPPs. However, the electric and magnetic field amplitudes of SPP standing waves change periodically along SPP propagation direction. As there is a difference of $\pi / 2$ between the amplitudes of $E_{x}^{\prime}$ and $E_{z}^{\prime}$, the maximum amplitude positions of $E_{x}^{\prime}$ corresponds to the minimum amplitude positions of $E_{z}^{\prime}$, and the antinode positions of electric field depend on the prominent z-component electric field $E_{z}^{\prime}$, satisfying $x=(2 n \pi+\pi-2 \Delta \varphi) / 2 k_{x}$, with $n=0, \pm 1, \pm 2 \cdots$. Thus, we can manipulate the antinode positions of SPP standing waves by changing the relative phase $\Delta \varphi$ between two excitations. Distances between two consecutive antinodes (refers to the maximum amplitude position) or nodes (refers to the minimum amplitude position) is $\lambda_{s p p} / 2$, in which $\lambda_{s p p}=2 \pi / k_{0} \sqrt{\left[\operatorname{Re}\left(\varepsilon_{m}\right)+\varepsilon_{2}\right] / \operatorname{Re}\left(\varepsilon_{m}\right) \varepsilon_{2}}$ is the excitation wavelength of SPPs. If the SPPs and SPP standing waves are excited by the same total incident power, e.g. $I_{0}=2 I_{1}$, the electric field intensity of antinodes is $2 \operatorname{Re}\left(\varepsilon_{m}\right) /\left[\operatorname{Re}\left(\varepsilon_{m}\right)+\varepsilon_{2}\right]$ times to the intensity of the SPPs. As the SPP intensity is limited by the number of free-electrons in metal surface, SPP standing waves can increase the localized SPP field intensity by modulating the free-electrons distribution.

We also use a FDTD solver from Lumerical Inc. to simulate the characteristics of SPP standing waves. In the simulation, the SPP standing waves are excited by a three-layer model consisting of a $50 \mathrm{~nm}$ Au film sandwiched between semi-infinite glass and air region, as shown in fig. 1. The dielectric constants of Au and glass are set as $\varepsilon_{m}=-10.8097+$ $0.7896 \mathrm{i}$ [22] and $\varepsilon_{1}=3.24$ at $633 \mathrm{~nm}$, respectively. The air-Au interface is set to be $z=$ $0 \mathrm{~nm}$. Two coherent TM plane waves illuminate to the glass-Au interface with opposite directions under the same incident angles. The incident angle $\theta$ is chosen to be 35.83 degree to excite the SPPs at air-Au interface in our simulations. To ensure accuracy, a $2.5 \mathrm{~nm}$ step override mesh is used to cover the SPP region. And the perfectly matched layer (PML) is set as the boundary conditions. Figure 2 shows the simulated electric intensity distributions at $z=2.5 \mathrm{~nm}$ (fig. 2 (a)) and $y=0 \mathrm{~nm}$ (fig. 2 (b)). The electric field intensities in fig. 2 are 
normalized to the simulated SPP intensity at $z=2.5 \mathrm{~nm}$, which is obtained by removing an incident TM wave and increase the other incident power twofold. Evidently, a SPP standing wave with periodic intensity is excited, which shows consistence with the theoretical analysis in Eq. (2).
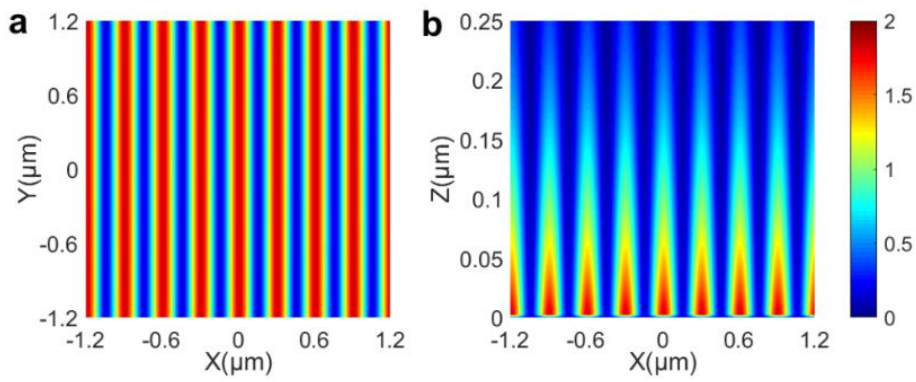

Fig. 2. The simulated electric intensity distributions of SPP standing waves at (a) $z=2.5 \mathrm{~nm}$ and (b) $y=0 \mathrm{~nm}$. The plots are normalized to the simulated SPP intensity distributions at $z=2.5 \mathrm{~nm}$ excited by the same total incident power.

\section{SPP standing waves interacting with single nanoparticles}

Due to the specific characteristics of SPP standing waves, some interesting feathers are obtained from the SPP standing waves interacting with single nanoparticles compared with SPPs. We situate a polystyrene (PS) nanoparticle with diameter being $100 \mathrm{~nm}$ on the air-Au interface at the position $(0,0,0)$. Figure 3 shows the simulated electric field intensity distributions at $z=2.5 \mathrm{~nm}$ of SPPs (in fig. 3 (a)) and SPP standing waves (in fig. 3 (b)) that interacts with the PS nanoparticles. The simulated electric field intensities in fig. 3 are subtracted by the same simulation without nanoparticles $[4,5,6,10]$ and normalized to the simulated SPP intensity distributions at $z=2.5 \mathrm{~nm}$. In SPP fields, the nanoparticle is polarized and generates localized enhancement near the particle. Meanwhile, single nanoparticles also reradiate SPP scattering, which interferes with launching SPPs and generates interference fringes. Figure 3 (b) shows the electric field intensity distribution of SPP standing waves interacting with single nanoparticles at $z=2.5 \mathrm{~nm}$. The relative phase of two excitations is $\Delta \varphi=\pi / 2$, and the particle is situated at the antinode of SPP standing waves. Similar with SPPs, both localized enhancement and interference fringes are also illustrated in SPP standing waves. Due to the symmetrical excitation of SPP standing waves, the symmetrical interference fringes are generated.
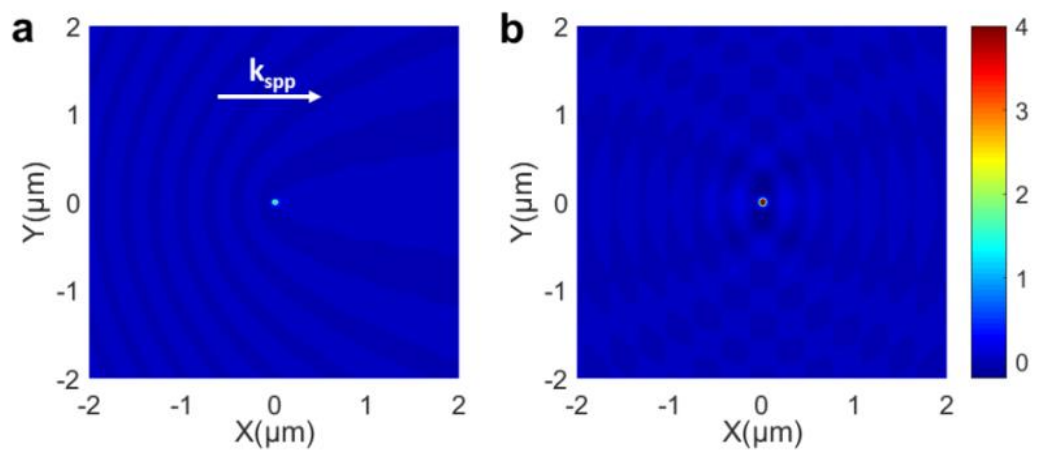

Fig. 3. Simulated electric field intensity distributions at $z=2.5 \mathrm{~nm}$ of (a) SPPs, and (b) SPP standing waves interact with PS particle of diameter being $100 \mathrm{~nm}$. The particle in (b) is located at the antinodes. The white arrow indicates the SPP propagation direction.

\section{Localized enhancements of SPP standing waves}


As the localized enhancement is critical to evaluate the sensitivity of label-free detection to single nanoparticles, we focus on analyzing the tailored localized enhancement of SPP standing waves. Figure 4 shows the near-field electric field intensity distribution of SPPs and SPP standing waves interacting with a PS particle with diameter being $60 \mathrm{~nm}$ at $y=$ $0 \mathrm{~nm}$ and $z=2.5 \mathrm{~nm}$. The nanoparticle is situated at the air-Au interface with $(0,0,0)$. Consistent with previous analysis, the electric field intensity distribution at $z=2.5 \mathrm{~nm}$ are subtracted by the same simulation without nanoparticles $[4,5,6,10]$. The dashed red line manifests the electric field intensity distribution along $x$-axis, and the solid blue line indicates the intensity distributions of incident SPPs and SPP standing waves, for simplicity, we call it incident intensity in this work. The black dashed lines depict the diameter of particle. In figs. 4 (a) - (c), the particle is polarized by the SPPs, charges accumulate around the top and the bottom of the particle and produce a vertical electric dipole [21]. The nanoparticle dipole couples with metal film, forms a nanocavity within the gap between particle and metal film, and produces a circular shaped localized enhancement at the air-Au interface (in fig. 4 (b)) $[23,24]$. As setting the monitor at $z=2.5 \mathrm{~nm}$ leads to part of monitors inside the nanoparticle, a dark region in center position appears. In figs. 4 (d) - (l), we simulate the electric field intensities of SPP standing waves interacting with single nanoparticles. Due to the periodic intensity of SPP standing waves, the nanoparticle polarizations can be tailored by changing the antinodes/nodes positions via manipulating the relative phase of two excitations $\Delta \varphi=\left(\varphi_{1}-\varphi_{2}\right) / 2$. In figs. 4 (d) - (f), we set the relative phase to be $\Delta \varphi=\pi / 2$ and obtain the electric field intensity distribution with the nanoparticles locating at antinodes. The polarized nanoparticle also acts as a vertical electric dipole, and the charge densities increase compared with those of SPPs. The charge accumulation with higher density near nanocavity brings the increased localized enhancement, leading to improved sensitivity for single nanoparticle detection, which will be discussed in details in section 5. In figs. 4 (j) - (1), the relative phase is set to be $\Delta \varphi=0$, and the nanoparticle is placed at the nodes. As the incident intensity at nodes is the weakest, more charges accumulate near both sides of the nanoparticle and the nanoparticle acts as a parallel dipole, leading to symmetrical localized enhancements. Owing to the low charge density near the nanocavity, the localized enhancement of particle situating at nodes is much weaker compared with nanoparticles located at other positions. For nanoparticle placed between antinodes and nodes, both vertical dipole and parallel dipole are excited. Asymmetric distributed localized enhancements are produced because of coupling of vertical and parallel electric dipoles. As shown in figs. $4(\mathrm{~g})-(\mathrm{i})$, the relative phase is $\Delta \varphi=\pi / 4$ and the nanoparticle is placed at the midpoint between antinodes and nodes. The intensity of forward scattering (relative to the SPP propagation direction) is increased while the backward scattering is decreased by the coupling between vertical and parallel dipoles, leading to an asymmetric localized enhancement. According to the above analysis, SPP standing waves interacting with small nanoparticles illustrates following properties: compared with vertical dipole in SPPs, both vertical dipole and parallel dipole can be excited in the SPP standing waves, the dipole modes can be controlled by changing particle center positions, i.e. closer to the antinode, the intensity of vertical dipole is stronger, and the parallel dipole is weaker. For the nanoparticles locating at antinodes, only the vertical dipole is excited and the localized enhancement is significantly increased. 


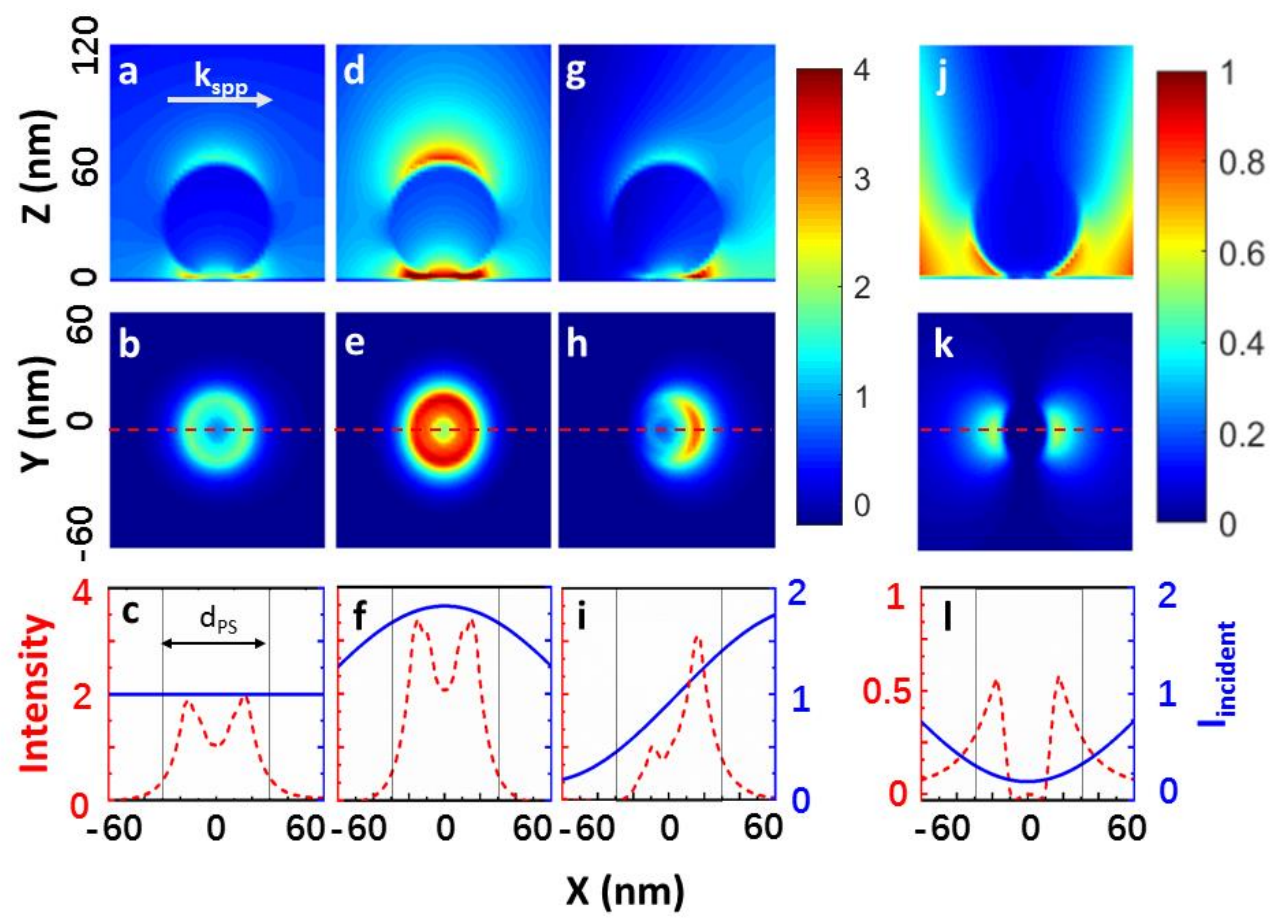

Fig. 4. Near-filed electric field intensity distributions of (a) - (c) SPPs and (d) - (1) SPP standing waves interact with a PS particle of diameter being $60 \mathrm{~nm}$. The nanoparticle is located at (d) - (f) antinode, (g) - (i) the midpoint between antinode and node, and (j) - (l) node, respectively. From top to bottom: the electric field intensity at top: $y=0 \mathrm{~nm}$, middle: $z=2.5 \mathrm{~nm}$, and bottom: intensity distributions (dashed red line) and incident intensities (solid blue line) along the $x$-axis with $z=2.5 \mathrm{~nm}$. The nanoparticle diameter $d_{p s}$ is depicted in black dashed lines.

Also, with increasing nanoparticle diameters comparable with incident wavelength, particle polarization exhibits directional properties [25], and the incident intensity of SPP standing waves changes significantly inside the particle, which influences the charge distributions. Figure 5 shows the near-field electric field intensity distributions induced by a PS particle with diameter being $300 \mathrm{~nm}$. As the particle diameter is larger than the decay depth of SPPs, charges densities around the top of the particle are lower than that of particle with diameter being $60 \mathrm{~nm}$, and charges mainly accumulates around the bottom of the particle. In figs. 5 (a) - (c), for SPPs, with particle diameter comparable with incident wavelength, the phase shift between opposite sides of particles induces the parallel dipole excitation. The coupling between vertical and parallel dipoles causes the directional particle polarization, leading to a stronger forward scattering and an asymmetric localized enhancement. For SPP standing waves, charge distributions are affected by the varied incident intensity. In figs. 5 (d) - (f), for nanoparticle placed in the antinodes with the relative phase $\Delta \varphi=\pi / 2$, the incident intensity on both sides of the particle is weak, which decreases the parallel polarization. The nanoparticle still acts as a vertical electric dipole, producing a circular shaped localized enhancement near the nanoparticle-metal nanocavity. In figs. 5 (j) - (1), with the particle locating in the nodes with $\Delta \varphi=0$, the incident intensities at both sides of the particle are strong, which increases the parallel polarization and decreases the vertical polarization. Thus, the nanoparticle acts as a parallel dipole, leading to symmetrical localized enhancements. For nanoparticle placed between antinodes and nodes, the coupling between vertical and parallel dipoles depends on varied incident intensity, leading to asymmetric localized enhancements. Thus, for SPP standing waves interacting with large nanoparticles of diameter comparable with incident wavelength, the 
polarization modes can also be controlled by changing the distance between particle center and antinodes.
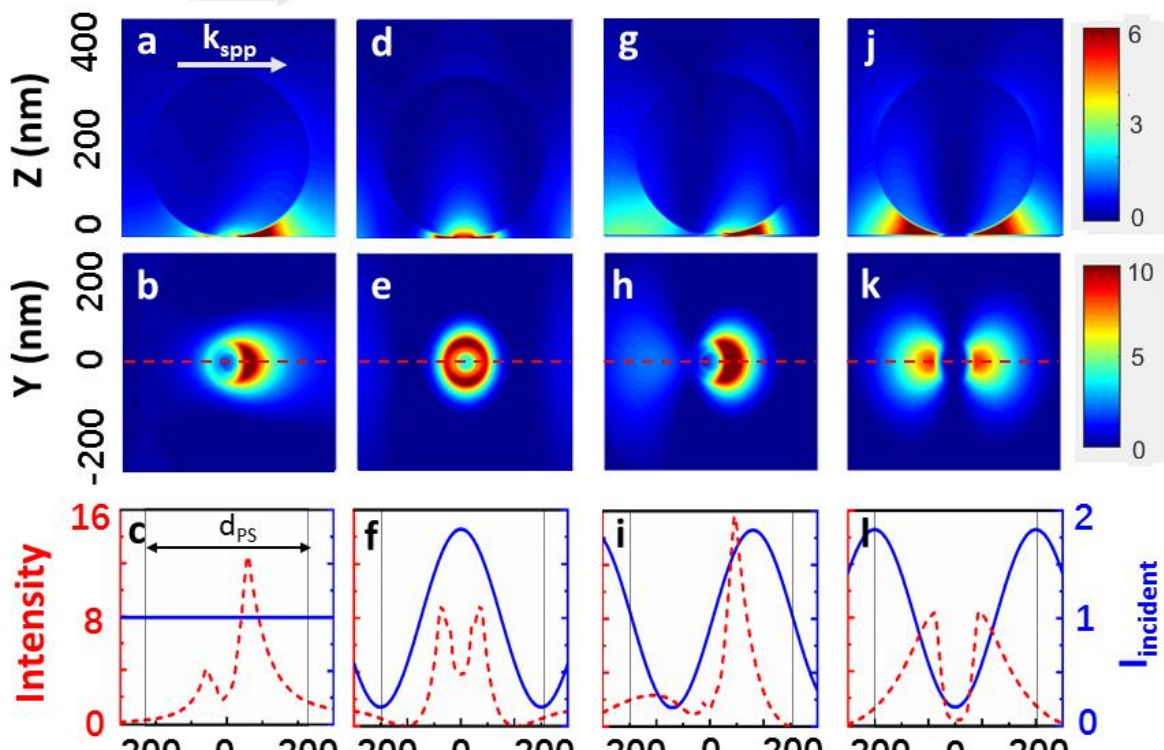

\section{$X(\mathrm{~nm})$}

Fig. 5. Near-filed electric field intensity distributions in (a) - (c) SPPs and (d) - (1) SPP standing waves interact with a PS particle of diameter being $300 \mathrm{~nm}$. The nanoparticle is located at (d) - (f) antinode, (g) - (i) the midpoint between antinode and node, and (j) - (1) node, respectively. From top to bottom row: the electric field intensity at top: $y=0 \mathrm{~nm}$, middle: $z=2.5 \mathrm{~nm}$, and bottom: intensity distribution (dashed red line) and incident intensity (solid blue line) along the x-axis with $z=2.5 \mathrm{~nm}$. The nanoparticle diameter $d_{p s}$ is depicted in black dashed lines.

\section{Comparison of localized enhancement between SPP standing waves and SPPs}

In this section, we discuss the influencing factors to localized enhancement of SPP standing waves induced by nanoparticles situating at antinode. Here we analyze the localized enhancement using localized enhancement intensity $I_{\text {sum }}$, which is obtained by counting the electric field intensities around the enhancement domain at $z=2.5 \mathrm{~nm}$ plane. Obviously, particle diameter is an important parameter that affects localized enhancement. Figure 6 shows the localized enhancement intensities of SPPs (in blue short dashed line) and SPP standing waves (in black dashed line) interacting with single PS nanoparticles with different diameters. The ratio of localized enhancement intensities between SPP standing waves and SPPs is also depicted in red solid line. In fig. 6 (a), the nanoparticles are placed at air-metal interface, and the distance between two consecutive antinodes is $302.5 \mathrm{~nm}$. Consistent with previous results depicted in Ref. 4 and 6, the localized enhancement intensities of SPPs increase nearly lineally with particle diameters. For SPP standing waves, the localized enhancement intensities increase with particle diameter being smaller than 220 $\mathrm{nm}$ and decrease with particle diameters changing from 220 to $300 \mathrm{~nm}$. The nonlinear localized enhancement intensity is originated from two reasons. Firstly, due to the inhomogeneous field distribution of SPP standing waves along $x$ axis, the incident intensity near nanocavity varies nonlinearly with particle diameter. Secondly, as discussed in section 3, the parallel dipole, which contributes to localized enhancement intensity in SPPs interacting with big nanoparticles, is not excited for nanoparticles at the antinode of SPP standing waves. Thus, the ratio of localized enhancement intensities between SPP standing 
waves and SPPs decreases with increasing nanoparticle diameter. For example, localized enhancement of SPP standing waves increases to 1.86 times compared to SPPs for single PS particle with diameter being $40 \mathrm{~nm}$. This increased localized enhancement is owing to higher charge densities of both metal film and polarized nanoparticle near the nanocavity.

The dielectric constant of surrounding medium also influences the localized enhancement. Figure 6 (b) shows the localized enhancement intensities of PS nanoparticles situating at Au-water interface. The incident angle $\theta$ is chosen to be 54.12 degree to excite the SPPs at Au-water interface, and distance between two consecutive antinodes is $217.5 \mathrm{~nm}$. Obviously, the SPP standing waves also indicate increased localized enhancement for small nanoparticles compared with SPPs. However, the improved localized enhancement is inferior compared with that of Au-air interface. As discussed in section 2, the ratio of electric field intensities between antinodes of SPP standing waves and SPPs is equal to $2 \operatorname{Re}\left(\varepsilon_{m}\right) /\left[\operatorname{Re}\left(\varepsilon_{m}\right)+\varepsilon_{2}\right]$. The increased $\varepsilon_{2}$ introduces a smaller intensity ratio between SPP standing waves and SPPs, leading to a decreased localized enhancement ratio. Meanwhile, the ratio of localized enhancement intensities between SPP standing waves and SPPs increases with nanoparticle diameter ranging from 260 to $300 \mathrm{~nm}$. The reason is that the period of SPP standing waves decreases with $\varepsilon_{2}$ and the incident intensity increases evidently with particle diameter in this region.
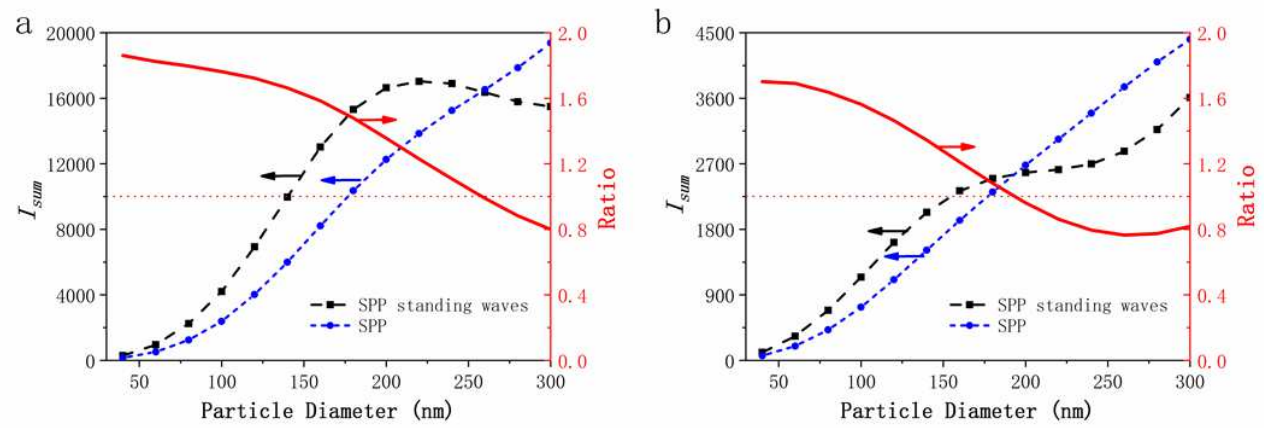

Fig. 6. Relationship between localized enhancement intensity and particle diameter with SPP standing waves (black dashed line), and SPPs (blue short dashed line). The ratio of localized enhancement intensities between SPP standing waves and SPPs is shown in red solid line. These nanoparticles are situated on (a) Au-air interface, and (b) Au-water interface. The particles are located at the antinodes of SPP standing waves.

We also investigate the localized enhancement intensities of nanoparticles with different refractive indexes situated at Au-air interface. Figure 7 shows the localized enhancement intensities of single nanoparticles with diameter being $60 \mathrm{~nm}$ interacting with SPPs and SPP standing waves, respectively. As the polarization charge densities increase with the refractive index of the nanoparticles, the localized enhancement intensities in both situations increase with particle refractive index. The ratio of localized enhancement intensities between SPP standing waves and SPPs is also depicted in red solid line, which decreases slowly with the increasing particle refractive index. The reason is that the intensity of parallel dipole increases with increasing particle refractive index in SPPs, which is not excited in SPP standing waves. According to above analysis, we conclude that the localized enhancement of SPP standing waves is tailored by particle diameter, dielectric constant of surrounding medium, and particle refractive index. Compared with SPPs, smaller particle size, lower dielectric constant of surrounding medium, and lower particle refractive index contribute to more increased localized enhancement of SPP standing waves. 


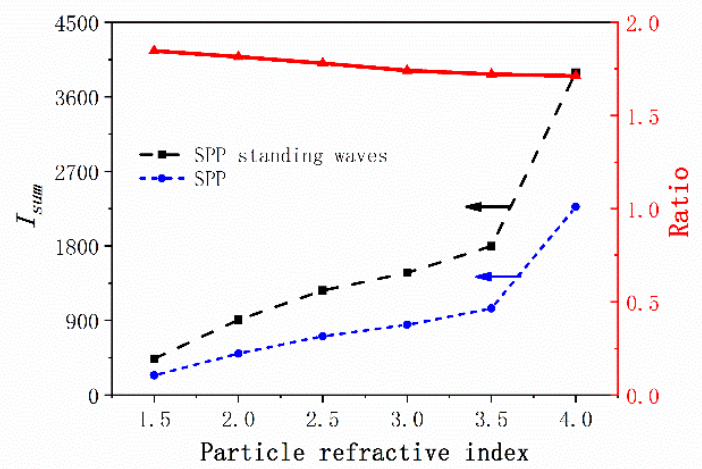

Fig. 7. Relationship between localized enhancement intensity and particle refractive index with particle diameter being $60 \mathrm{~nm}$ interacting with SPP standing waves (black dashed line), and SPPs (blue short dashed line) at Au-air interface. The ratio of localized enhancement intensities between SPP standing waves and SPPs are shown in red solid line. These nanoparticles are situated at the antinodes of SPP standing waves.

\section{Summary}

In this work, we explore the localized enhancements of SPP standing waves interacting with single nanoparticles. The periodic intensity distribution of SPP standing waves provides varied incident fields by manipulating the relative phase of two SPP excitations, which controls the electric dipole modes of the nanoparticle and tailors the localized enhancements. The vertical and parallel electric dipoles are excited by situating nanoparticles at antinode and node, respectively, and both parallel and vertical electric dipoles are excited at other positions. For nanoparticles at antinodes, high-density charges around nanoparticle-metal nanocavity are induced, leading to enhanced near-field localized intensity, which is more evidently for small nanoparticles. The localized enhancement also connects with dielectric constant of surrounding medium and particle refractive index. Smaller dielectric constant of surrounding medium introduces larger periods and stronger incident intensity at antinodes, resulting in more increased localized enhancement. Larger particle refractive index induces higher intensity of parallel dipole in SPPs and diminishes the ratio of localized enhancements. As the localized enhancement connects to the sensitivity of label-free detection to single nanoparticles, the SPP microscopy with higher sensitivity can be realized by using SPP standing waves. In addition, the symmetric distribution of localized enhancements of SPP standing waves at antinodes and nodes make it easy to find the center of nanoparticles, which provides a potential method for highprecision positioning to single nanoparticles.

\section{Declarations}

Funding Information This works is partly supported by the National Key Research and Development Program of China grant numbers 2017YFF0107002, 2018YFC2001100 and 2020YFC2004503; Beijing Natural Science Foundation grant numbers 4192063 and 4182073; Scientific Research Equipment Project of Chinese Academy of Sciences under Grant No. YJKYYQ20190056; Guangzhou Science and Technology Project grant number 201604020005; Guangdong Province Science and Technology Project grant number 2016A040403086; State Key Joint Laboratory of Environment Simulation and Pollution Control grant number 18K07ESPCT.

Conflict of Interest The authors declare no conflicts of interest. 
Availability of data and material The data that support the findings of this study are available from the corresponding author upon reasonable request.

Code availability Not applicable.

Author Contribution All the authors contributed in the preparation of manuscript. The article has been read and approved for submission by all the authors.

Ethics approval Not applicable.

Consent to participate Not applicable.

Consent for publication Not applicable.

Acknowledgments We thank Dr. Wei Xiong from Institute of Microelectronics of Chinese Academy of Sciences for his useful discussion.

\section{References}

1. Narimanov E (2019) Resolution limit of label-free far-field microscopy, Advance Photonics 1:056003. https://doi.org/10.1117/1.AP.1.5.056003

2. Maier S A (2007) Plasmonics: Fundamentals and Applications. Springer US, New York

3. Zhang X, Xu Q, Xia L, Li Y, Gu J, Tian Z, Ouyang C, Han J, Zhang W (2020) Terahertz surface plasmonic waves: a review. Advance Photonics 2: 014001. https://doi.org/10.1117/1.AP.2.1.014001

4. Zybin A, Kuritsyn Y A, Gurevich E L, Temchura V V, Überla K, Niemax K, (2010) Real-time Detection of Single Immobilized Nanoparticles by Surface Plasmon Resonance Imaging. Plasmonics 5:31-35. https://doi.org/10.1007/s11468-009-9111-5

5. Wang W (2018) Imaging the chemical activity of single nanoparticles with optical microscopy. Chem. Soc. Rev. 47: 2485-2508. https://doi.org/10.1039/C7CS00451F

6. Sun X, Liu H, Jiang L, Wei R, Wang X, Wang C, Lu X, Huang C (2019) Detecting a single nanoparticle by imaging the localized enhancement and interference of surface plasmon polaritons. Opt Lett 44: 5707-5710. https://doi.org/10.1364/OL.44.005707

7. Li Z, Fang Y, Wang Y, Jiang Y, Liu T, W Wang (2017) Visualizing the bidirectional electron transfer in a Schottky junction consisting of single CdS nanoparticles and a planar gold film. Chem Sci 8: 5019-5023. https://doi.org/10.1039/C7SC00990A

8. Chen J, Zhou K, Wang Y, Gao J, Yuan T, Pang J, Tang S, Chen H, Wang W (2019) Measuring the activation energy barrier for the nucleation of single nanosized vapor bubbles. PNAS 116: 12678-12683. https://doi.org/ 10.1073/pnas.1903259116

9. Liu S, Zhou K, Yuan T, Lei W, H Chen, Wang X, Wang W (2020) Imaging the Thermal Hysteresis of Single Spin-Crossover Nanoparticles. J Am Chem Soc 142: 15852-15859. https://doi.org/10.1021/jacs.0c05951

10. Viitala L, Maley A M, Fung H W M, Corn R M, Viitala T, Murtomäki L (2016) Surface Plasmon Resonance Imaging Microscopy of Liposomes and Liposome-Encapsulated Gold Nanoparticles. J Phys Chem C 120: 25958-25966. https://doi.org/10.1021/acs.jpcc.6b09503

11. Wang S, Shan X, Patel U, Huang X, Lu J, Li J, Tao N (2010) Label-Free Imaging, Detection, and Mass Measurement of Single Viruses by Surface Plasmon Resonance. PNAS 107: 16028-32. https://doi.org/10.1073/pnas.1005264107

12. Sun X, Liu H, Yang Y, Xiong W, Chen Y, Jiang L, Li N, Lu X, Tang H, Xia Y (2017) Imaging to single virus by using surface plasmon polariton scattering. Proc of SPIE 10244:1024425. https://doi.org/10.1117/12.2264434

13. Grave P L, Domínguez D (2013) SPP tomography experiments with surface plasmon polariton standing waves. Opt Commun 286:151-155. https://doi.org/10.1016/j.optcom.2012.08.061

14. Cheng Q Q, Li T, Guo R Y, Li L, Wang S M, Zhu S N (2012) Direct observation of guided-mode interference in polymer-loaded plasmonic waveguide. Appl Phys Lett 101: 171116. https://doi.org/10.1063/1.4764116

15. Day J K, Large N, Nordlander P, Halas N J(2015) Standing Wave Plasmon Modes Interact in an AntennaCoupled Nanowire. Nano Lett 15:1324-1330. https://doi.org/10.1021/n15045428

16. Chung E, Kim Y, Tang W T, Sheppard C J R, Peter T C S (2009) Wide-field extended-resolution fluorescence microscopy with standing surface-plasmon-resonance waves. Opt Lett 34: 2366-2368. https://doi.org/10.1364/ol.34.002366

17. Wei F, Liu Z (2010) Plasmonic Structured Illumination Microscopy. Nano Lett 10: 2531-2536. https://doi.org/10.1021/nl1011068

18. Meng F, Du L, Yang A, Zhang C, Yuan X (2019) Low-loss metal-dielectric waveguide mode enabled structured illumination microscopy with $0.18 \lambda_{0}$ resolution. Opt Express 27: 9250-9257. https://doi.org/10.1364/OE.27.009250 
19. Angela D, Alexei A K (2015) Principles of nanoparticle imaging using surface plasmons. New J Phy 17: 013041. https://doi.org/10.1088/1367-2630/17/1/013041

20. Søndergaard T, Bozhevolnyi S I (2004) Surface plasmon polariton scattering by a small particle placed near a metal surface, An analytical study. Phys Rev B 69: 045422. https://doi.org/10.1103/PhysRevB.69.045422

21. Evlyukhin A B, Reinhardt C, Evlyukhin E, Chichkov B N (2013) Multipole analysis of light scattering by arbitrary-shaped nanoparticles on a plane surface. J Opt Soc Am B 30: 2589-2598. https://doi.org/10.1364/josab.30.002589

22. Babar S, Weaver J H (2015) Optical constants of Cu, Ag, and Au revisited. Appl Opt 54: 477-481. https://doi.org/10.1364/AO.54.000477

23. Evlyukhin A B, Bozhevolnyi S I (2015) Resonant unidirectional and elastic scattering of surface plasmon polaritons by high refractive index dielectric nanoparticles. Phys Rev B 92: 245419. https://doi.org/10.1103/PhysRevB.92.245419

24. Li G, Zhang Q, Maier S, Lei D (2018) Plasmonic particle-on-film nanocavities: A versatile platform for plasmon-enhanced spectroscopy and photochemistry. Nanophotonics 7: 1865-1889. https://doi.org/10.1515/nanoph-2018-0162

25. Mie G (1908) Beitrage zur Optik trüber Medien, speziell kolloidaler Metallosungen. Leipzig Ann Phys 330: 377-445. https://doi.org/10.1002/andp.19083300302 


\section{Figures}

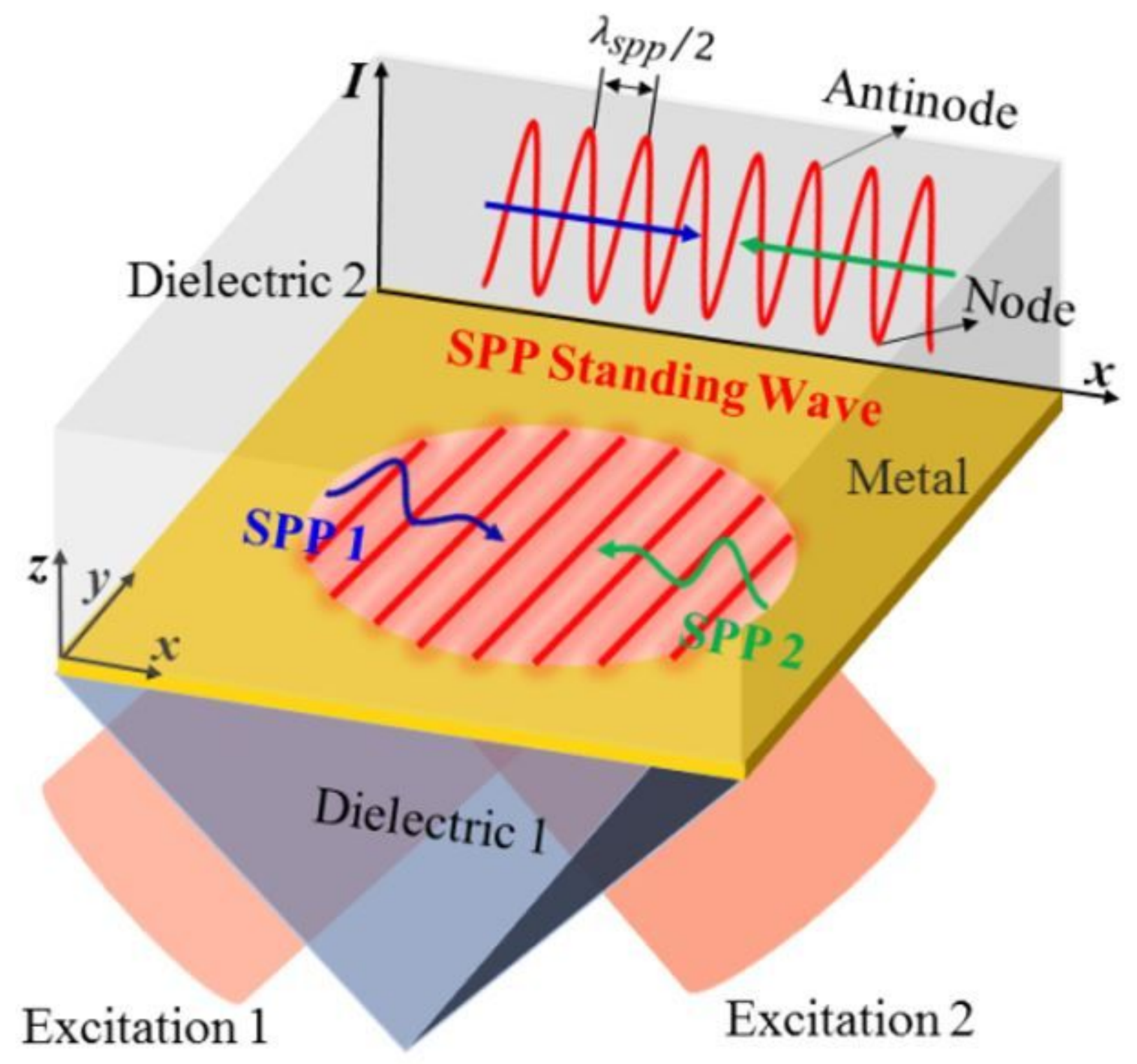

Figure 1

The model of SPP standing wave excitation in Kretschmman configuration with dielectric-metal-dielectric system 

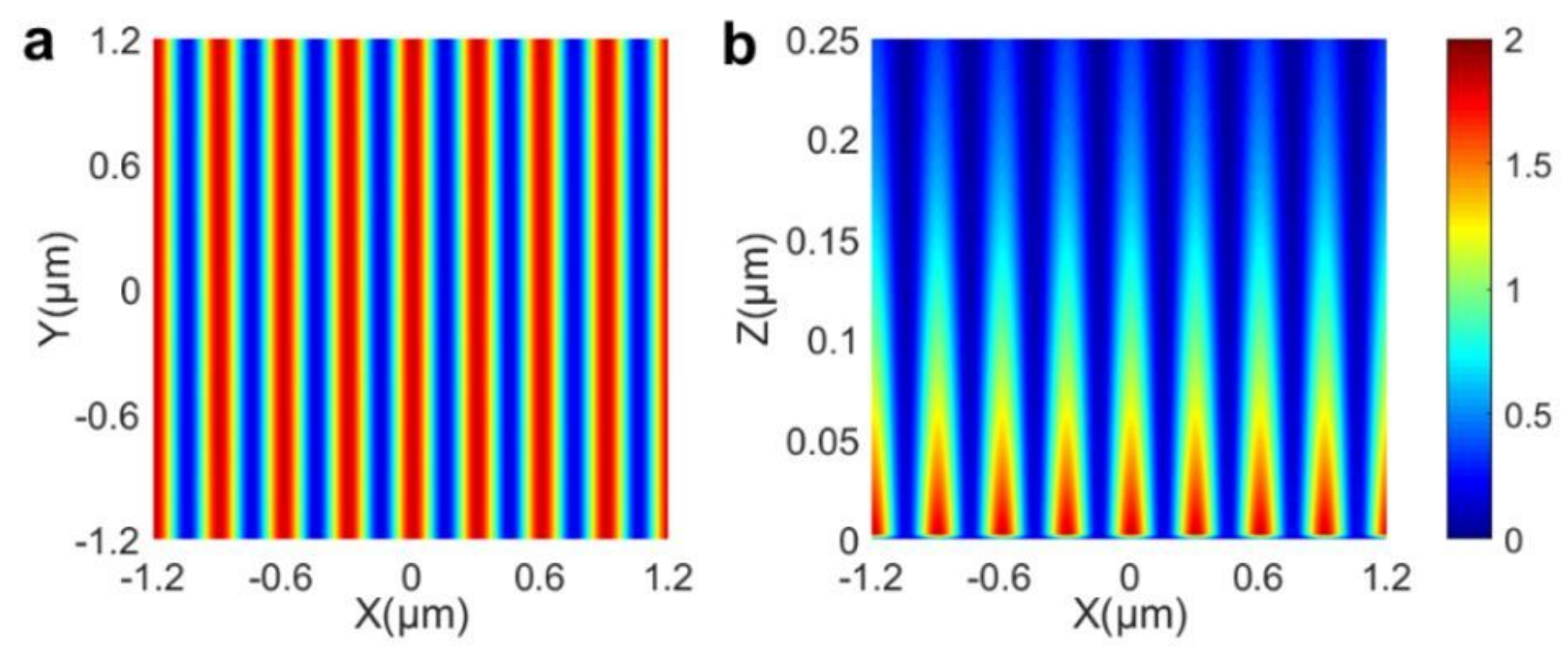

Figure 2

The simulated electric intensity distributions of SPP standing waves at (a) $z=2.5 \mathrm{~nm}$ and (b) $y=0 \mathrm{~nm}$. The plots are normalized to the simulated SPP intensity distributions at $\mathrm{z}=2.5 \mathrm{~nm}$ excited by the same total incident power.
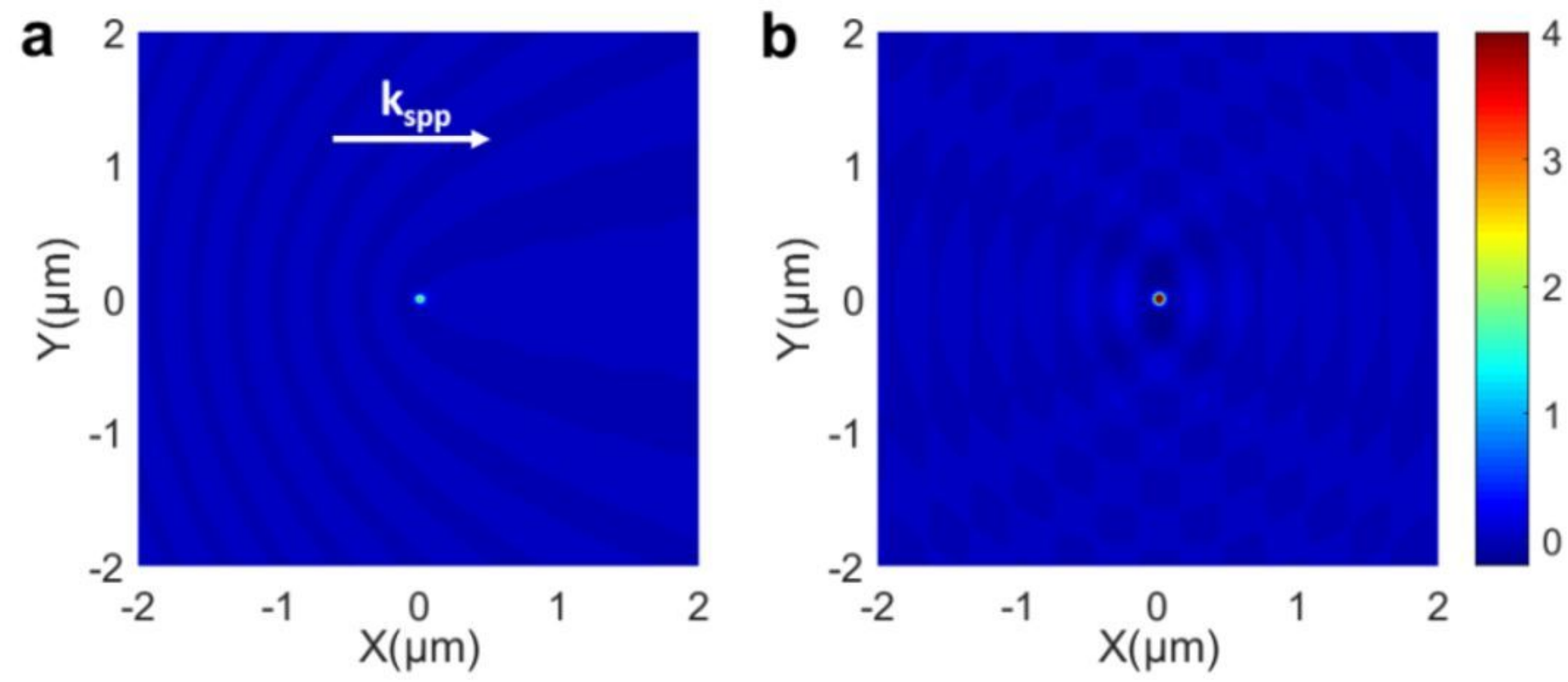

\section{Figure 3}

Simulated electric field intensity distributions at $z=2.5 \mathrm{~nm}$ of (a) SPPs, and (b) SPP standing waves interact with PS particle of diameter being $100 \mathrm{~nm}$. The particle in (b) is located at the antinodes. The white arrow indicates the SPP propagation direction. 


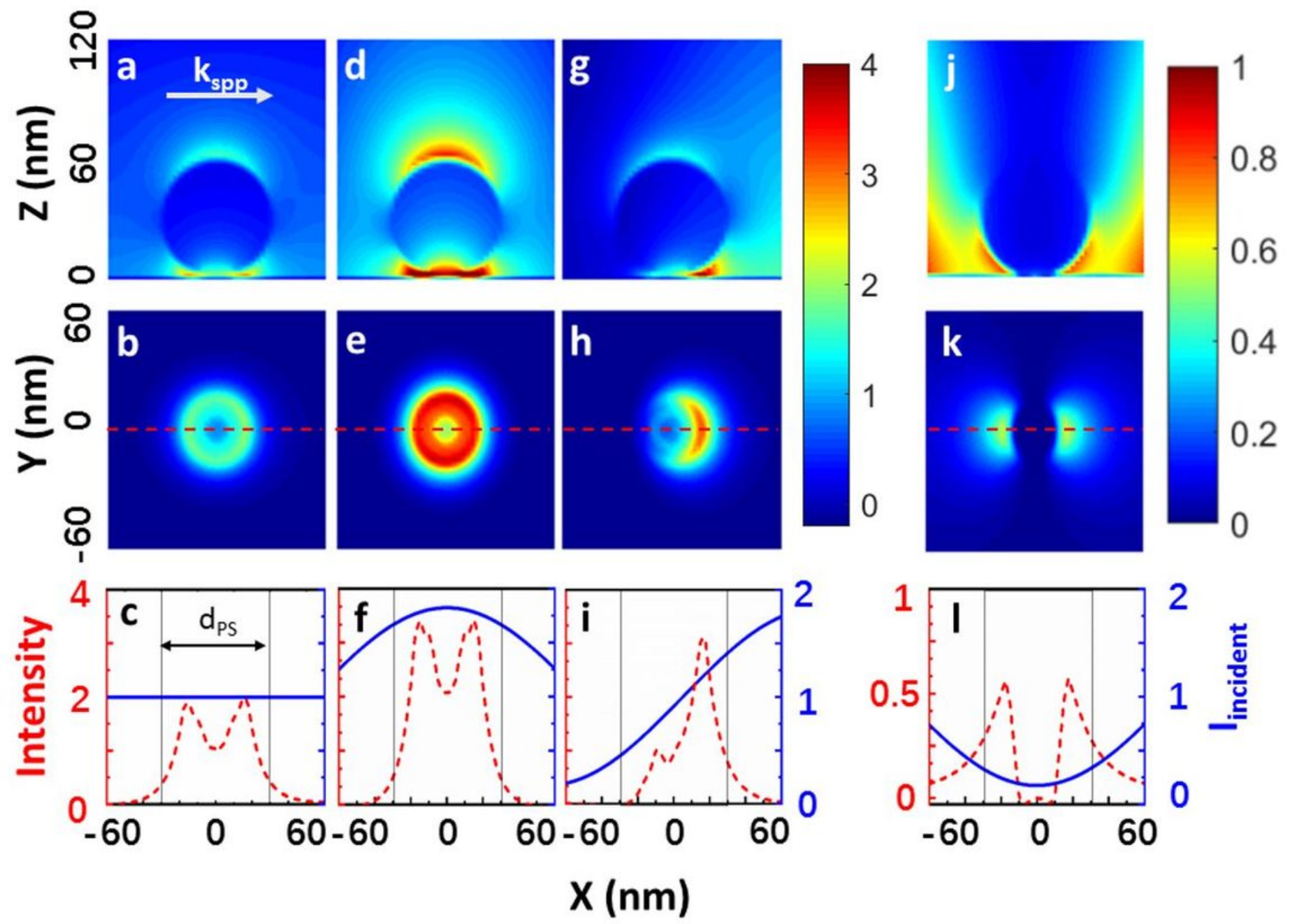

Figure 4

Near-filed electric field intensity distributions of (a) - (c) SPPs and (d) - (I) SPP standing waves interact with a PS particle of diameter being $60 \mathrm{~nm}$. The nanoparticle is located at (d) - (f) antinode, (g) - (i) the midpoint between antinode and node, and (j) - (I) node, respectively. From top to bottom: the electric field intensity at top: $y=0 \mathrm{~nm}$, middle: $\mathrm{z}=2.5 \mathrm{~nm}$, and bottom: intensity distributions (dashed red line) and incident intensities (solid blue line) along the $x$-axis with $z=2.5 \mathrm{~nm}$. The nanoparticle diameter dps is depicted in black dashed lines. 


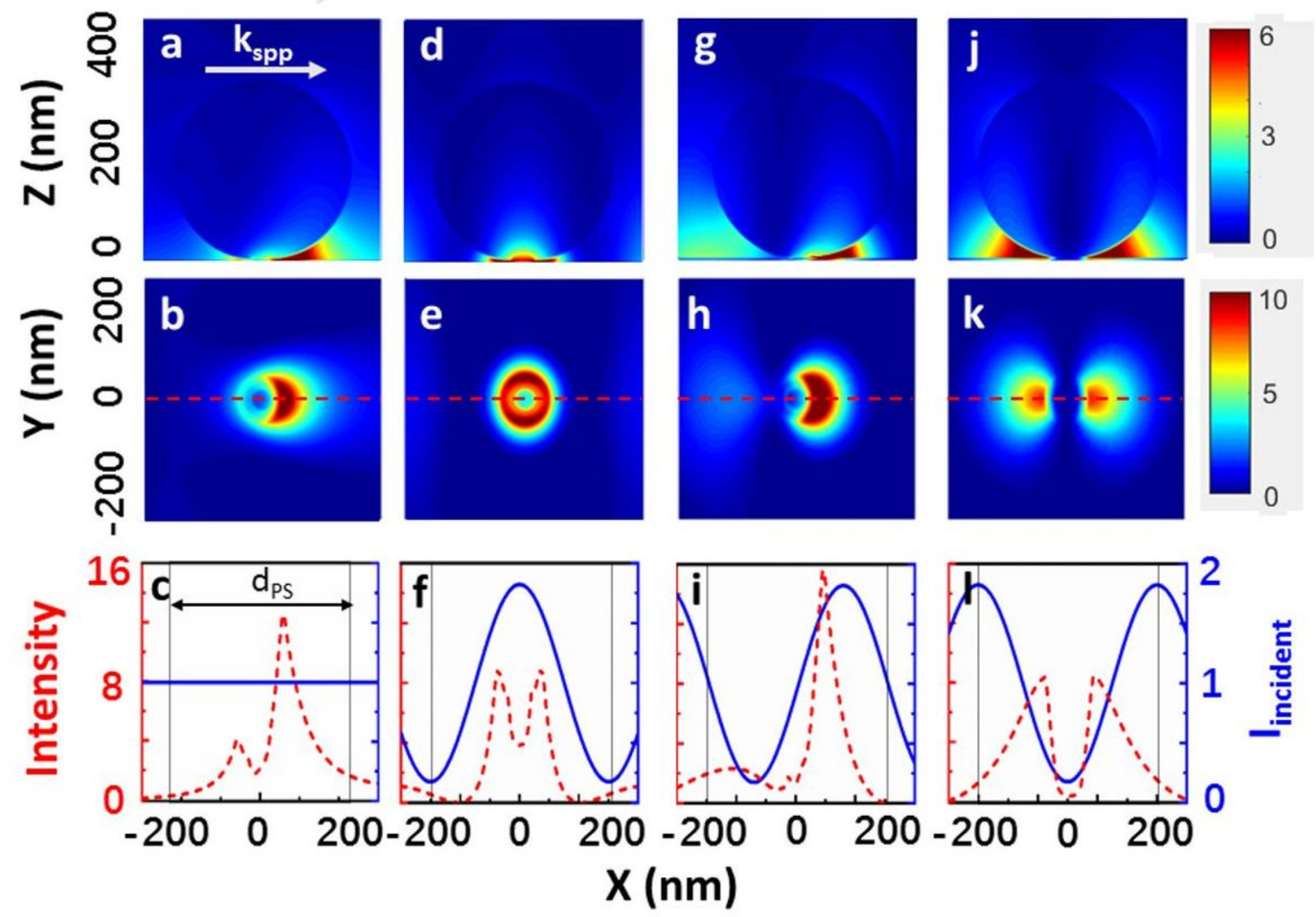

Figure 5

Near-filed electric field intensity distributions in (a) - (c) SPPs and (d) - (I) SPP standing waves interact with a PS particle of diameter being $300 \mathrm{~nm}$. The nanoparticle is located at (d) - (f) antinode, (g) - (i) the midpoint between antinode and node, and (j) - (I) node, respectively. From top to bottom row: the electric field intensity at top; $y=0 \mathrm{~nm}$, middle: $\mathrm{z}=2.5 \mathrm{~nm}$ and bottom: intensity distribution (dashed red line) and incident intensity (solid blue line) along the $x$-axis with $z=2.5 \mathrm{~nm}$. Teh nanoparticle dps is depicted in black dashed lines. 

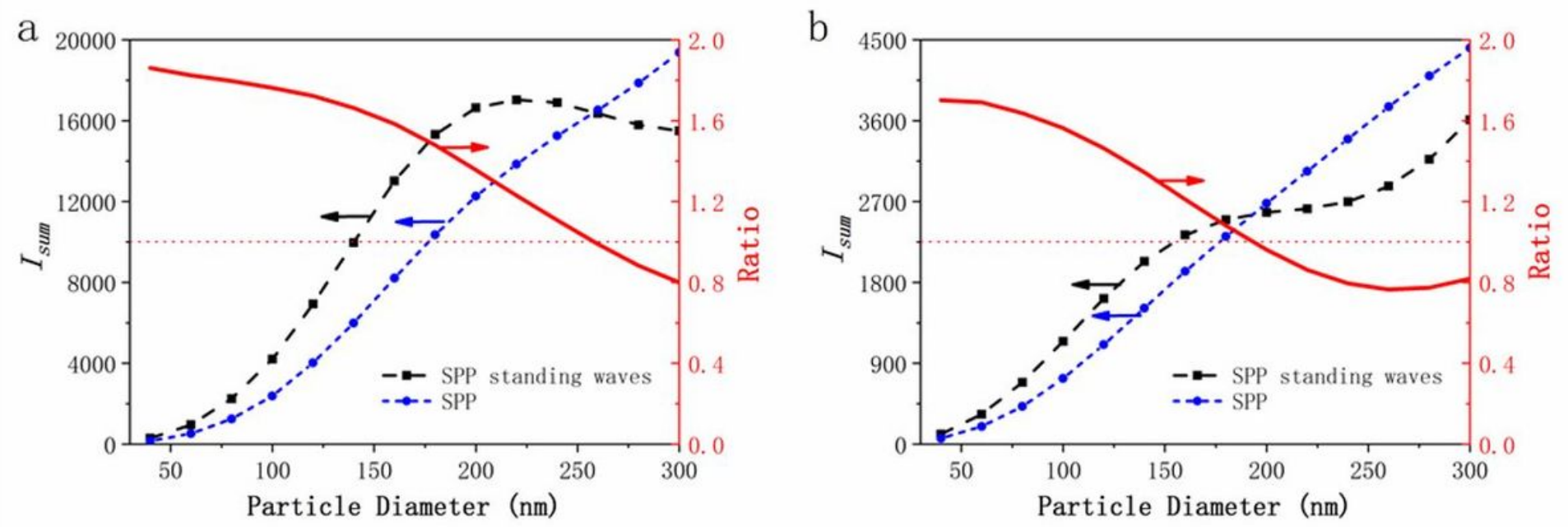

Figure 6

Relationship between localized enhancement intensity and particle diameter with SPP standing waves (black dashed line), and SPPs (blue short dashed line). The ratio of localized enhancement intensities between SPP standing waves and SPPs is shown in red solid line. These nanoparticles are situated on (a) Au-air interface, and (b) Au-water interface. The particles are located at the antinodes of SPP standing waves.

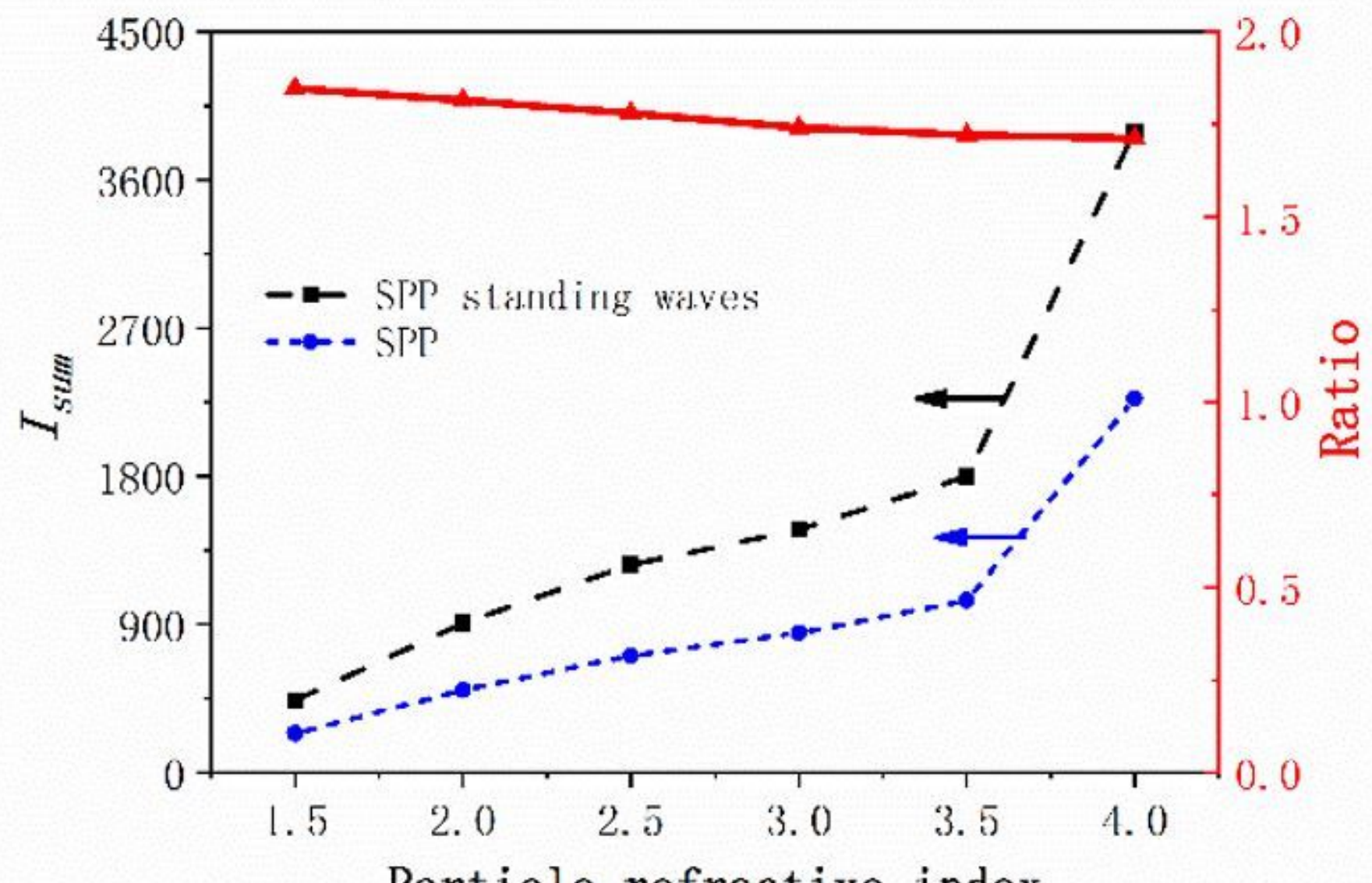

Figure 7 
Relationship between localized enhancement intensity and particle refractive index with particle diameter being $60 \mathrm{~nm}$ interacting with SPP standing waves (black dashed line), and SPPs (blue short dashed line) at Au-air interface. The ratio of localized enhancement intensities between SPP standing waves and SPPs are shown in red solid line. These nanoparticles are situated at the antinodes of SPP standing waves. 